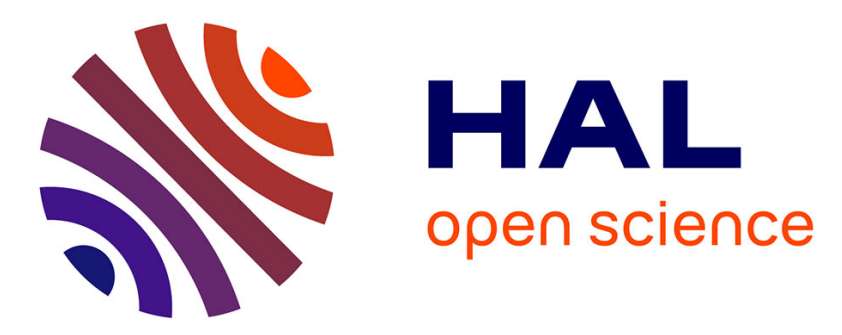

\title{
Response of wall hot-film gages with longitudinal diffusion and conduction to the substrate
}

Sedat F. Tardu, C.Thanh Pham

\section{To cite this version:}

Sedat F. Tardu, C.Thanh Pham. Response of wall hot-film gages with longitudinal diffusion and conduction to the substrate. Journal of Heat Transfer, 2005, 127, pp.812-819. 10.1115/1.1928907 . hal-00262010

\section{HAL Id: hal-00262010 https://hal.science/hal-00262010}

Submitted on 25 Feb 2020

HAL is a multi-disciplinary open access archive for the deposit and dissemination of scientific research documents, whether they are published or not. The documents may come from teaching and research institutions in France or abroad, or from public or private research centers.
L'archive ouverte pluridisciplinaire HAL, est destinée au dépôt et à la diffusion de documents scientifiques de niveau recherche, publiés ou non, émanant des établissements d'enseignement et de recherche français ou étrangers, des laboratoires publics ou privés. 


\section{Response of Wall Hot-Film Gages With Longitudinal Diffusion and Heat Conduction to the Substrate}

\begin{abstract}
The effects of heat transfer into a substrate and axial diffusion are analyzed through numerical simulations in order to elucidate the frequency response of wall hot-film gages. The ratio of the conductivities of the substrate and fluid plays an important role in steady flows when it is larger than 5 and the Péclet number is smaller than 50 . The equivalent film length increases considerably with the conductivity ratio, and it decreases when a thin film of low conductivity is sandwiched between the hot-film gage and the substrate, showing a net improvement of the heat transfer conditions. The frequency response in unsteady flows is highly attenuated in the presence of heat transfer into the substrate. The cutoff frequency is strongly dependent on the conductivity ratio. Improved response is obtained with the two-layer substrate configuration. It is further shown that axial diffu-sion considerably affects the frequency response when the shear and/or the streamwise length of the film are small.
\end{abstract}

Keywords: Hot-Film Gauge, Axial Diffusion, Conduction to the Substrate, Frequency Response, Full 2D Simulations

\section{Introduction}

The wall shear stress is a fundamental quantity in near-wall turbulent flows. It provides a rapid diagnostic of the flow and is essential in drag reduction problems, which are of great industrial importance. The suboptimal or optimal control of drag requires, however, a highly dense distribution of the actuators and wall shear stress sensors [1]. The streamwise and spanwise spacing of these MEMS (micro-electromechanical systems) have to be as small as the viscous sublayer thickness to obtain a drag reduction of about $20 \%$. That means that the largest dimension of the shear stress sensors used for control purposes has to be smaller than about $100 \mu \mathrm{m}$ even at moderate Reynolds numbers. This miniaturization poses problems, because some effects that are neglected under conventional situations have to be thoroughly taken into account in the physical mechanisms governing the sensor under these circumstances.

The direct measurements of wall shear stress turbulent fluctuations are still not feasible [2]. The classical indirect measurements are based either on heat (thermo-anemometry) or mass (electrochemical-anemometry) transfer techniques. The latter presents some advantages caused by an absence of the parasitical effects of the substrate, but it can only be used in some liquid flows. Thus development of measurement techniques of shear stress through hot-film gages is an ongoing challenge, especially with regard to the MEMS sensors design [3].

Complete analytical solutions of the full problem governing heat transfer over the wall hot-film (WHF) do not exist for the following reasons.

i. The indirect heat transfer from the hot film to the substrate by conduction, and the subsequent transfer from the substrate to the fluid and its interaction with the convective direct heat transfer are not explicitly known.

ii. The coupling of the effect of the longitudinal diffusion through the trailing and leading edge singularities with the unsteady response of the thermal boundary layer increases the complexity of the problem. Longitudinal diffusion plays an important role when the streamwise length of the film and/or the shear is small. The boundary layer approximation in the convective heat transfer equation is not valid under these circumstances. This effect may be more severe for MEMS micro-sensors and severely decrease the frequency response.

There are many studies available in the literature on the thermal boundary layer characteristics induced by a hot-film gauge (HFG) flush mounted at the wall. Several theoretical, numerical, and experimental studies have been reported in the past since the classical Lévêque solution of the thermal boundary layer problem over a finite strip maintained at a constant temperature. The validity of the boundary layer approximation is questioned in the numerical analysis conducted by Ling [4], and later in the theoretical approach of Springer and Pedley [5] and Springer [6]. These authors have shown that axial diffusion may be predominant at small Péclet numbers, i.e., either when the streamwise length of the film or the shear stress is small. The perturbation analysis conducted by Ackerberg et al. [7] has clearly indicated that the Lévêque solution is valid only for Péclet numbers larger than roughly 100. Their theoretical analysis is in good agreement with measurements obtained by electrochemical probes.

Lighthill [8] was first to investigate the unsteady response of a thermal boundary layer over a plate maintained at constant temperature. He showed that the thermal inertia decreases the unsteady response in the high-frequency regime. Bellhouse and Schultz [9] analyzed the influence of the fluctuating wall shear stress on the frequency response of a hot film by using Lighthill's technique with a 1D model. They showed the importance of the substrate conductivity on the frequency response. Brison et al. [10] studied the same problem in a $2 \mathrm{D}$ configuration. They used a quasi-steady forced convection law and ignored axial diffusion. The conjugated heat transfer problem was examined by Cole and Beck [11] and Moen and Schneider [12] without the effect of axial diffusion.

The present investigation studies the combined effects of axial diffusion through the trailing and leading edges of the HFG and conduction to the substrate on the frequency response of the wall 

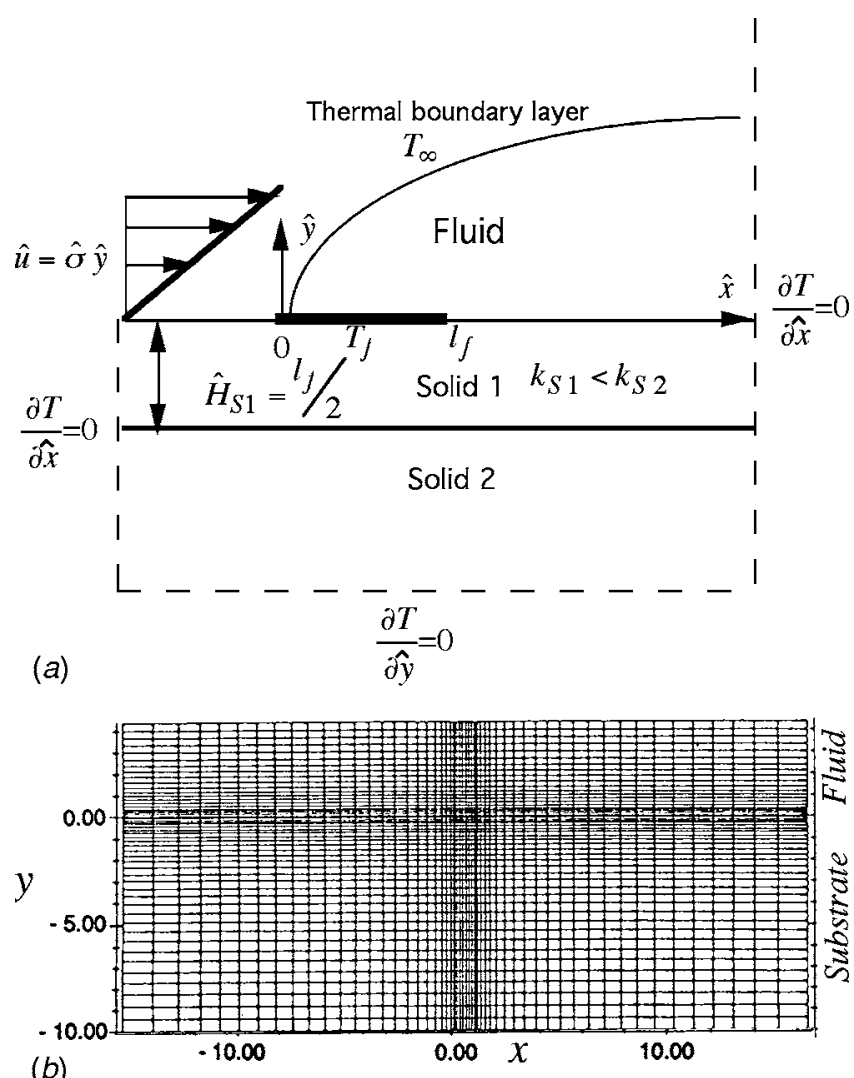

Fig. 1 (a) Model with a two-layer substrate and the corresponding boundary conditions. (b) Typical computational domain and mesh distribution.

shear stress sensors. The full 2D formulation of the conjugate heat transfer problem is presented here, and the results are analyzed both in the steady and unsteady regimes.

\section{Formulation of the Problem and the Numerical Method}

The dimensionless equations in the fluid $F$, in the solid $S$, and at the fluid-solid (substrate) $F-S$ interface are respectively

$$
\begin{gathered}
F: \quad \frac{\partial \theta}{\partial t}+u \frac{\partial \theta}{\partial x}=\frac{\partial^{2} \theta}{\partial x^{2}}+\frac{\partial^{2} \theta}{\partial y^{2}} \\
S: \quad \frac{\alpha_{F}}{\alpha_{S}} \frac{\partial \theta}{\partial t}=\frac{\partial^{2} \theta}{\partial x^{2}}+\frac{\partial^{2} \theta}{\partial y^{2}} \\
F-S: \quad \frac{k_{F}}{k_{S}} \frac{\partial \theta_{F}}{\partial y}=\frac{\partial \theta_{S}}{\partial y}
\end{gathered}
$$

The boundary conditions are $\partial \theta / \partial x \rightarrow 0$, and $\partial \theta / \partial y \rightarrow 0$, as $x, y$ $\rightarrow \infty$, in the substrate and $\partial \theta / \partial x \rightarrow 0$ for $x \rightarrow \pm \infty$, and $\theta \rightarrow 0$ when $y \rightarrow \infty$ in the fluid. The velocity distribution in the forced convection heat transfer equation is either $u(y)=\sigma y$ or $u(y, t)=\sigma y(1$ $\left.+a \sin \omega^{*} t\right)$ depending on whether a steady or unsteady problem is studied. The velocity is linear since the thermal boundary layer is thin, exactly as in the classical Lévêque's problem. The shear parameter is given by $\sigma=\operatorname{Pr}\left(l_{f} u_{\tau} / \nu\right)^{2}=\operatorname{PrRe}_{f}^{2}$.

The sizes of the computational domain are $70 l_{f}$ in the $x$ direction, as well as $6 l_{f}$ in the fluid and $20 l_{f}$ in the substrate in the $y$ direction. It was carefully checked that these dimensions are large enough to guarantee the validity of the boundary conditions. Figure $1(a)$ shows schematically the computational domain and the boundary conditions for a two-layer substrate.
The heat transfer law generally used for wall shear stress measurements (the Nusselt number $\mathrm{Nu} \propto \sigma^{1 / 3}$ ) is based on the simplest solution which assumes $\partial \theta / \partial t=\partial^{2} \theta / \partial x^{2}=0$ in the fluid (quasisteadiness and no longitudinal diffusion) and $k_{S}=0$. More complete solutions have been considered in the past, for instance with $\partial \theta / \partial t \neq 0[13-15]$ or with $k_{S} \neq 0$ (in the 1D case by Bellhouse and Schultz [9] and the 2D case by Brison et al. [10]). The full simulation introduces the additional parameter of the diffusivity ratio $\alpha_{\mathrm{F}} / \alpha_{\mathrm{S}}$, which points to the importance of how fast the heat is transferred to the fluid after conduction through the solid in addition to how much heat is transferred this way as compared to the direct transfer on the film.

Upstream weighted differencing [16] and ADI (alternated direction implicit), combined with stretched coordinates [17], near the trailing and leading edges of the WHF, as well as near the wall have been used. The ADI is chosen because it is unconditionally stable and quite efficient in problems involving heat and mass transfer. The upstream weighted differencing scheme is essentially a control-volume approach. The temperatures on the control mesh are determined with unknown interpolation coefficients, which are given later as data for the computation. These coefficients depend on the problem studied and the mesh sizes of the computational space. The resulting equation is stable if all the coefficients are positive. The time step is chosen uniform over the whole range of the computational space. One of the advantages of this strategy is that the same code may be run for different cases, as for example the boundary layer approximation $\left(\partial^{2} \theta / \partial x^{2}=0\right)$, by simply setting the corresponding coefficients equal to zero. The numerical scheme is of second order in time and space.

The analysis of heat transfer near the singularities at the wall and trailing and leading edges requires a dense grid distribution. Stretching coordinates are used to avoid the numerical problems caused by a nonuniform grid distribution. The stretching function in the streamwise $x$ coordinate is $x(\xi)=\sin ^{2}(\pi / 2 \xi)$ for $0 \leqslant x, \xi$ $\leqslant 1$. The stretching is symmetrical with respect to the center of the hot film. The smallest grid size near the singularities is $\Delta x$ $=\sin ^{2}\left[\pi /(4 K)^{2}\right]$, where $K$ is the number of grid points on the film. One has $\Delta x \ll 1 / K^{2}$ for a moderate $K$. The same stretching approach is used upstream and downstream of the film, and it is matched with the coordinate system on the film. A typical computational domain is shown in Fig. 1(b). The number of computational nodes varied between $70 \times 50$ (in streamwise and wall normal directions, respectively) to typically $120 \times 150$.

The stretching method used in the fluid and substrate following the wall normal direction is of the form $y=\eta^{2}$. Thus for the model shown in Fig. 1(a), the coordinate transformations are

$$
\begin{gathered}
F: \quad 0 \leqslant y \leqslant H_{F}, \quad 0 \leqslant \eta \leqslant c_{F} H_{F} ; \quad y(\eta)=\eta^{2} /\left(c_{F}^{2} H_{F}\right) \\
S 1 \text { (solid 1): }-H_{S 1} \leqslant y \leqslant 0, \quad-c_{S 1} H_{S 1} \leqslant \eta \leqslant 0 ; \\
y(\eta)=-\eta^{2} /\left(c_{S 1}^{2} H_{S 1}\right) \\
S 2(\text { solid } 2):-\left(H_{S 1}+H_{S 2}\right) \leqslant y \leqslant-H_{S 1}, \\
-\left(c_{S 1} H_{S 1}+-c_{S 2} H_{S 2}\right) \leqslant \eta \leqslant-c_{S 1} H_{S 1} ; \\
y(\eta)=-\left(\eta+c_{S 1}^{2} H_{S 1}\right)^{2} /\left(c_{S 2}^{2} H_{S 2}\right)-H_{S 1}
\end{gathered}
$$

The coefficients $c_{F}, c_{S 1}$, and $c_{S 2}$ can be modified to adapt the stretching to different zones. The maximum resolution occurs at the interfaces where the derivative $d y / d \eta=0$. The upstream weighting difference and ADI problems are formulated in the $(\xi, \eta)$ domain and the resulting system was resolved by the classical Thomas algorithm.

Several computations were performed to check the performance of the numerical method. We first consider a steady flow on a perfectly isolated substrate. Figure 2 shows the local flux $q_{w}^{*}$ $=-(\partial \theta / \partial y)_{y=0} \sigma^{-1 / 3}$ over the film for two values of the shear pa- 


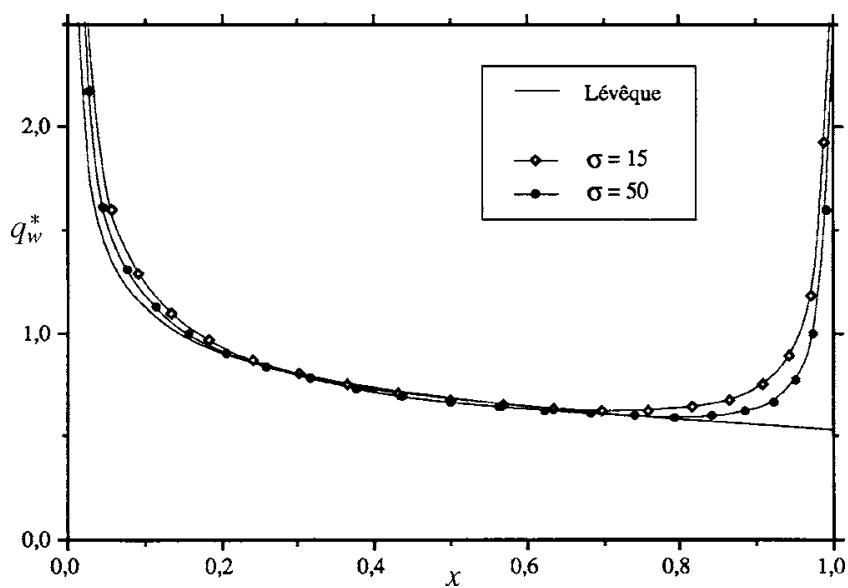

Fig. 2 Local heat flux over the film compared with the boundary layer approximation (Lévêque solution)

rameter. The effect of the trailing edge singularity increases with decreasing Péclet number. The computed global heat flux is compared with Ling [4] and Springer [6] in Fig. 3. There is an excellent agreement with the asymptotic analysis of Springer [6], and the difference between the computations and his theoretical approach is less than $1 \%$.

\section{Results}

3.1 Two-Layer Configuration; Homogeneous Substrate. The global heat transfer rates per unit span $Q$ for the coupled glass/water (conductivity ratio $\left.k_{S} / k_{F}=1.27\right)$ and glass/air $\left(k_{S} / k_{F}\right.$ $=25.3)$ cases are shown in Tables $1(a)$ and $1(b)$ as a function of the Péclet number $\sigma=\operatorname{Pr} l_{f}^{+2}$. The notations are illustrated in Fig. 4. In the steady regime, the heat transfer from the film to the substrate $Q_{f S}$ must be balanced by heat loss from the semi-infinite substrate to the fluid. Thus, $Q_{f S}=Q_{S F}^{-}+Q_{S F}^{+}$. This balance is, by the way, a good check for computational accuracy. The fluid is heated by the upstream heat transfer rate $Q_{S F}^{-}$before it reaches the film. This decreases both the direct transfer rate from the film $Q_{f F}$ and the downstream transfer rate $Q_{S F}^{+}$. The latter extends the heating of the fluid further downstream and smoothes the effect of the axial diffusion at the trailing edge singularity. The conductivity ratio $k_{S} / k_{F}$ is the only relevant parameter describing the substrate's direct and indirect influences. The direct transfer is reduced because of the wake effect due to $Q_{S F}^{-}$and the indirect transfer

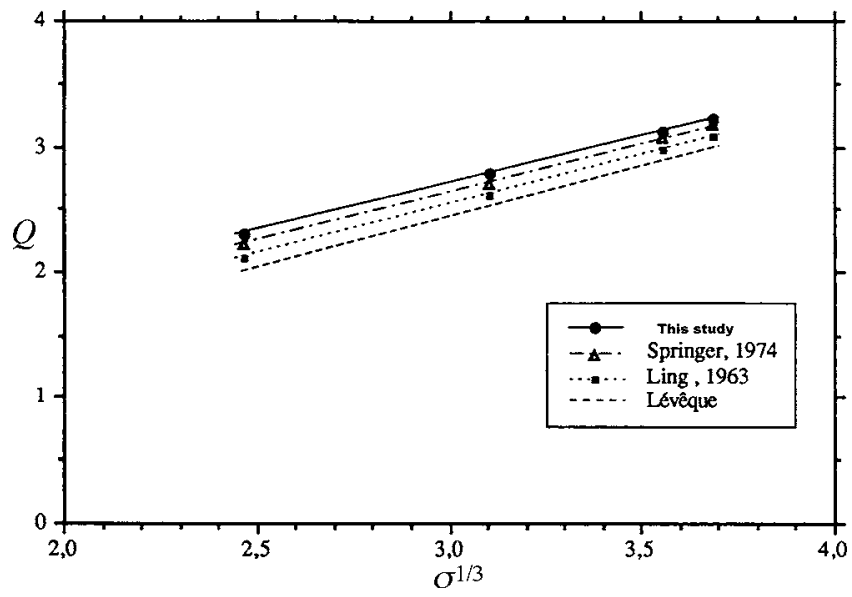

Fig. 3 Global heat flux with the longitudinal diffusion term and comparison with past studies
Table 1 Global heat transfer rates versus the shear parameter. For the notations see Fig. 4. (a) Water/glass. (b) Air/glass.

\begin{tabular}{c|c|c|c|c|c|c}
\hline \hline $\boldsymbol{\sigma}$ & $\mathbf{Q}$ & $\mathbf{Q}_{\text {fF }}$ & $\mathbf{Q}_{\mathrm{fS}}$ & $\mathbf{Q}_{\mathbf{S F}}^{-}$ & $\mathbf{Q}_{\mathbf{S}}^{+} \mathbf{F}$ & QLévêque \\
\hline 3 & 9,74 & 0,63 & 9,11 & 5,37 & 3,74 & 1,16 \\
\hline 15 & 12,79 & 1,05 & 11,74 & 7,37 & 4,37 & 1,99 \\
\hline 23 & 13,70 & 1,23 & 12,47 & 7,89 & 4,57 & 2,30 \\
\hline 30 & 14,00 & 1,34 & 12,66 & 8,05 & 4,60 & 2,51 \\
\hline 38 & 14,26 & 1,43 & 12,82 & 8,18 & 4,65 & 2,71 \\
\hline 45 & 14,39 & 1,50 & 12,89 & 8,23 & 4,66 & 2,87
\end{tabular}

together with the ratio $Q_{f S} / Q_{f F}$ increase with $k_{S} / k_{F}$.

The heat flux to the substrate $Q_{f S}$ is smaller than the direct transfer $Q_{f F}$ for small conductivity ratios as expected (Fig. 5(a)). On the contrary, the ratio $Q_{f S} / Q_{f F}$ is as large as 10 for the combination air-glass with $k_{S} / k_{F}=25.3$ as seen in Fig. $5(b)$. For both cases the ratio $Q_{f S} / Q_{f F}$ decreases with increasing Péclet number, because the transfer to the substrate $Q_{f S}$ depends only slightly upon $\sigma$, while the transfer from the film to the fluid $Q_{f F}$ increases much more rapidly with the Péclet number by forced convection.

For the combination water/glass, the direct transfer from the film to the fluid is approximately equal to the Lévêque solution,

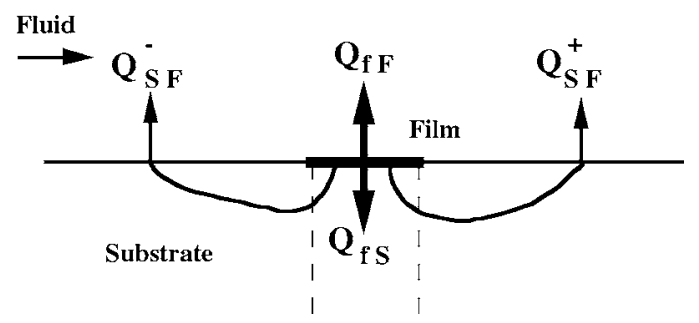

Fig. 4 Sketch of heat transfer definitions over the hot film gage
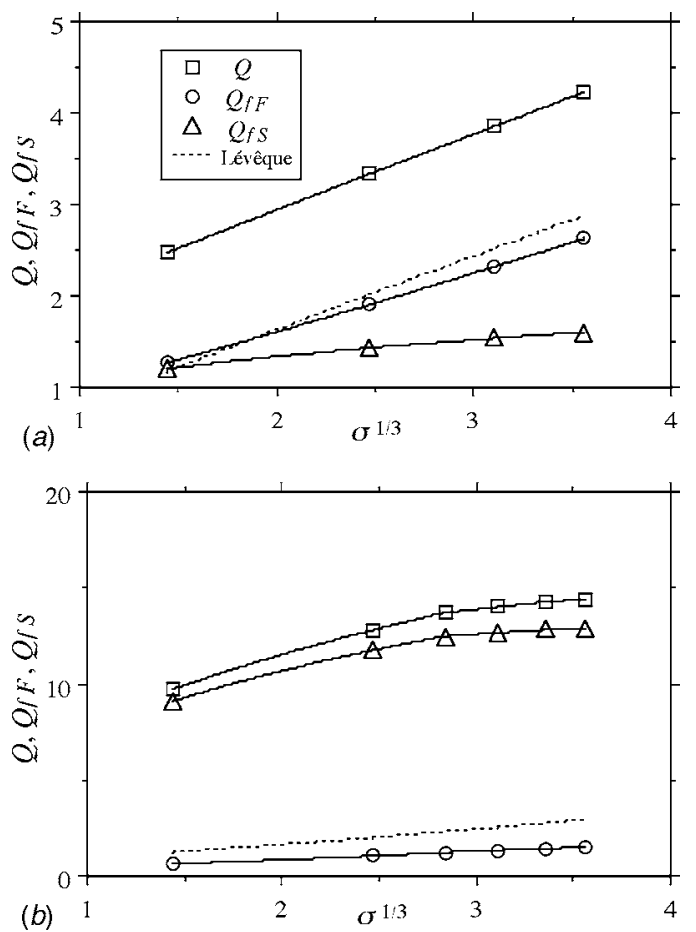

Fig. 5 Direct transfer to the fluid and transfer to the substrate compared with the Lévêque solution. (a) Water/glass. (b) Air/ glass. Legend on (a). 


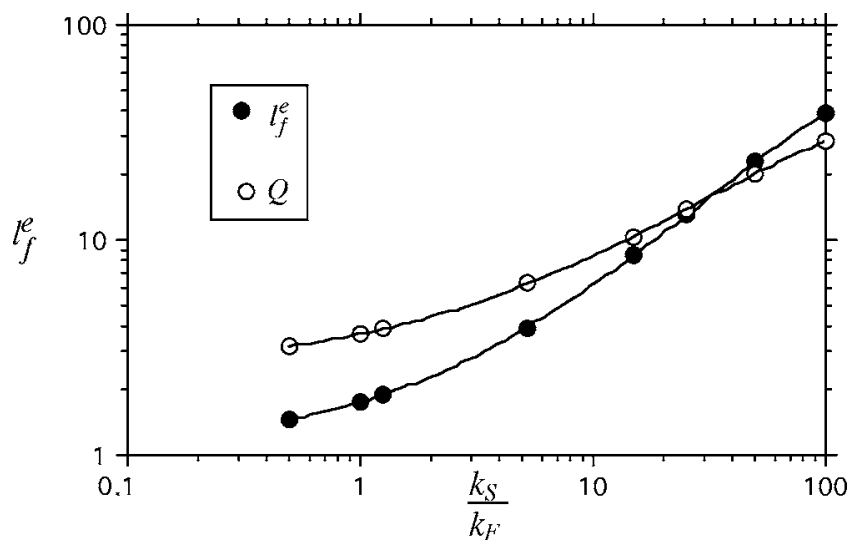

Fig. 6 Effective length of the hot film gage versus the conductivity ratio for $\sigma=30$

i.e., $Q_{f F} \approx Q_{\text {Lévêque }}=0.81 \sigma^{1 / 3}$ (Fig. 5(a)). For large $\sigma$ values, $Q_{f F}$ is smaller than $Q_{\text {Lévêque }}$, but the difference never exceeds $10 \%$.

3.2 Effect of the Conductivity Ratio. The effect of heat transfer via the substrate may be characterized by an "equivalent length" $l_{f}^{e}$ defined by $Q=Q_{f F}+Q_{f S}=0.81 \sigma^{1 / 3}\left(l_{f}^{e}\right)^{2 / 3}$. Hence, $l_{f}^{e}$ is the dimensionless length of a hypothetical film on a perfectly nonconducting substrate, obeying the Lévêque law giving the same total heat transfer as the real film. The impact of the ratio $k_{S} / k_{F}$ on the heat transfer process is further analyzed for a fixed Péclet number $\sigma=30$. Figure 6 shows that the effective length and the global transfer $Q$ increase considerably with the conductivity ratio. A regression analysis shows that

$$
Q=2.44\left(\frac{k_{S}}{k_{F}}\right)^{0.54} \text { and } l_{f}^{e}=0.96\left(\frac{k_{S}}{k_{F}}\right)^{0.81}
$$

in the range $5<k_{S} / k_{F}<100$. Hence, the effective length is 1.5 times more sensitive to the conductivity ratio than $Q$. The global flux depends strongly upon the conductivity ratio and varies approximately as the square root of $k_{s} / k_{F}$. These results show that the hot-film gauge can be applies to the mixtures such as heliumair, because the variations of heat transfer rates depend on the conductivity of the fluid, which is a function of the composition of the binary mixture. The increase of the global heat transfer rate is obviously due to the importance of heat conduction to the substrate $Q_{f S}$ at high conductivity ratios (Fig. $7(a)$ ). The upstream heat transfer rate $q_{S F}^{-}$is always larger than $Q_{S F}^{+}$and the difference $Q_{S F}^{-}-Q_{S F}^{+}$increase with $k_{s} / k_{F}$ (Fig. 7(b)). Consequently, the direct transfer is weakend by the wake effect caused by upstream transfer $Q_{S F}^{-}$. The ideal Lévêque solution is never exactly reached even at low conductivity ratios such as $k_{S} / k_{F} \approx 1$.

3.3 Two-Layer Substrate. Computations were also carried out for a two-layer substrate configuration, in which a thin isolating mylar film of thickness $0.5 l_{f}$ is sandwiched between the hot wall film and the substrate. Such a configuration exists commercially (DANTEC glue-on probe 55R47). The transfer depends on both $k_{S 1} / k_{F}$ (mylar-fluid) and $k_{S 2} / k_{F}$ (substrate-fluid). Two cases were studied, namely

(i) $k_{S 1} / k_{F}=1, k_{S 2} / k_{F}=5.2$ (helium-mylar-glass),

(ii) $k_{S 1} / k_{F}=4.9$, and $k_{S 2} / k_{F}=25.3$ (air-mylar-glass)

A low indirect transfer $Q_{f S}$ is expected in the first case, since the ratio $k_{S 1} / k_{F}$ is relatively small. Case (i) is indeed somewhat similar to the water-glass combination $\left(k_{S} / k_{F}=1.3\right)$. The equivalent film lengths are indeed approximately equal in these cases (Fig. 8 ). The indirect transfer is, however, $10 \%$ larger in case (i), compared to water-glass because of larger $k_{S 2} / k_{F}$ and this explains the
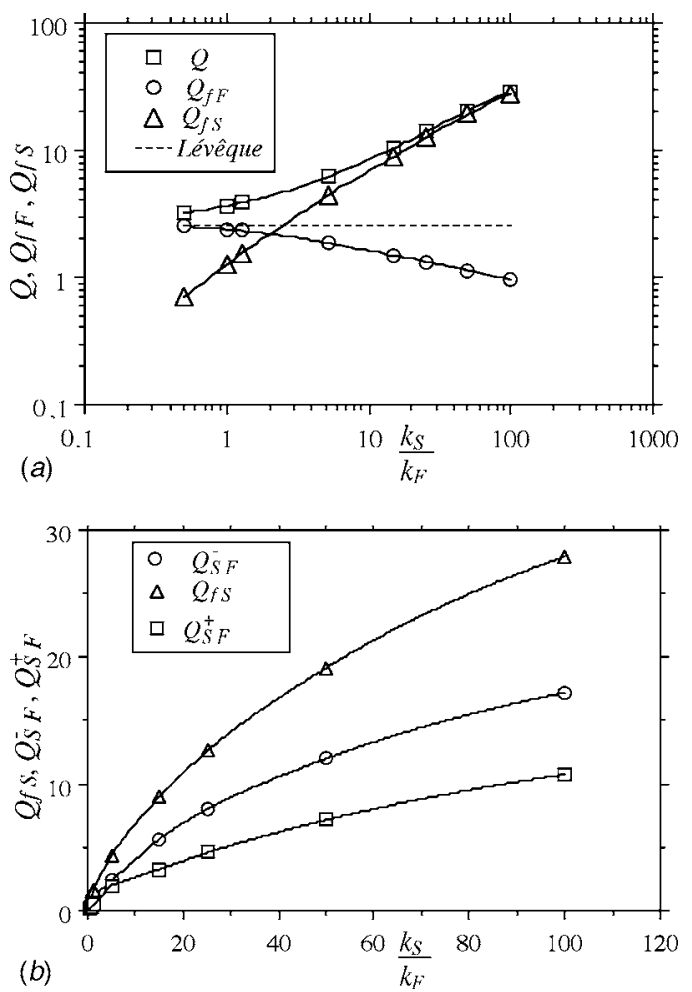

Fig. 7 Global heat transfer rates versus the conductivity ratio. (a) Transfer to the fluid and to the substrate. (b) Transfer rates from the substrate to the fluid upstream and downstream of the hot film.

slight differences observed in Fig. 8.

The case of air-mylar-glass is more interesting because it shows how the isolating thin film may improve the global performance of the hot film gauge. Thus, the effective length and the transfer to the substrate decrease by a factor of 2 , and the direct transfer $Q_{f F}$ is 30\% larger compared with the mono-layer glass substrate. Moreover, the relationship $Q=f\left(\sigma^{1 / 3}\right)$ tends rapidly to a Lévêque's type law $Q=A \sigma^{1 / 3}+B$ when the Péclet number is high enough, more rapidly with the isolating film than without. This is summarized in Fig. 9. The explanation of the decrease of the equivalent length with increasing velocity (or shear) and increasing values of the ratio $k_{F} / k_{S}$ lies in the increased capacity of the fluid to carry away the heat conducted through the solid or, in other words, in the capacity of the fluid to cool the solid substrate heated above ambient temperature in the vicinity of the film.

The temperature at the wall decreases rapidly in a zone of radius $l_{f}$ in the case of a double-layer substrate (Fig. 10). Figure 11

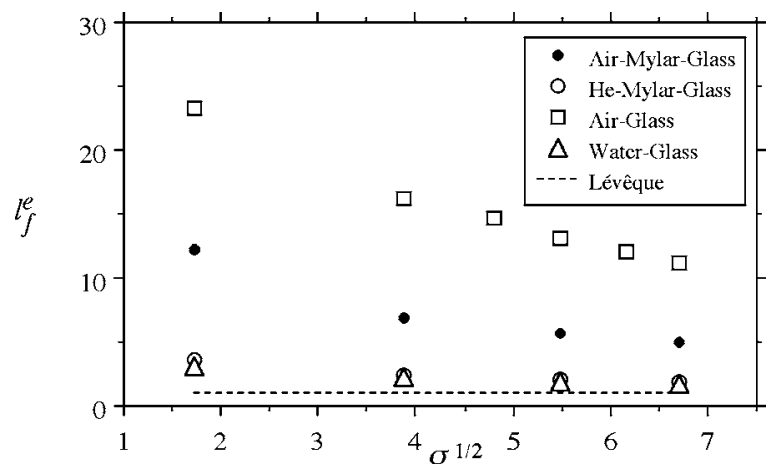

Fig. 8 The effective length of the hot film with a double-layer substrate 


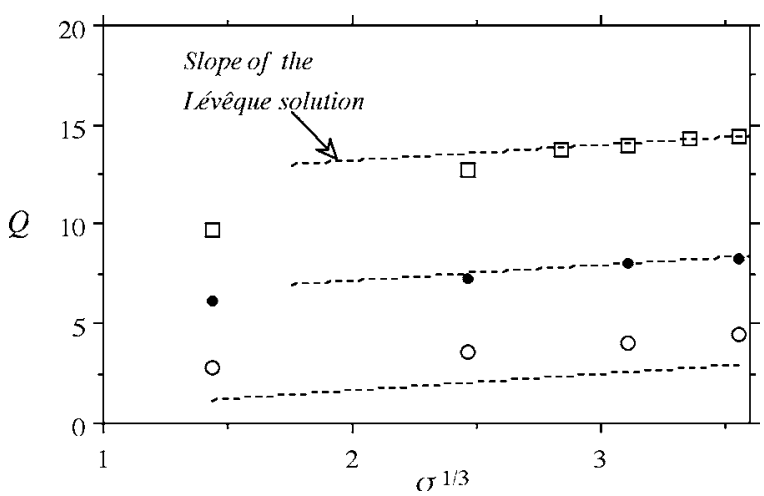

Fig. 9 Global heat flux for different substrate configurations and comparison with the Lévêque solution. For legend see Fig. 8.

shows that the local flux is also more important compared with the air-glass case. The heat flux at the trailing edge is two times larger when the substrate has a multi-layer structure.

3.4 Frequency Response. The frequency response of the hot film gauge is determined by imposing a sinusoidal shear parameter $\sigma=\bar{\sigma}(1+a \sin \omega t)$. The periodical variations of the direct and indirect transfer rates as a function of the imposed frequency $\omega^{*}$ are determined for different configurations. The unsteady computations were started from the time-mean steady field and were carried out until the difference in temperature at any point was less than $0.01 \%$ between two successive imposed periods. The dimensionless parameters governing the problem are $\bar{\sigma}, a, \omega^{*}$, $k_{S} / k_{F}, \alpha_{S} / \alpha_{F}, k_{S 1} / k_{S 2}$, and $\alpha_{S 1} / \alpha_{S 2}$ with the last two parameters

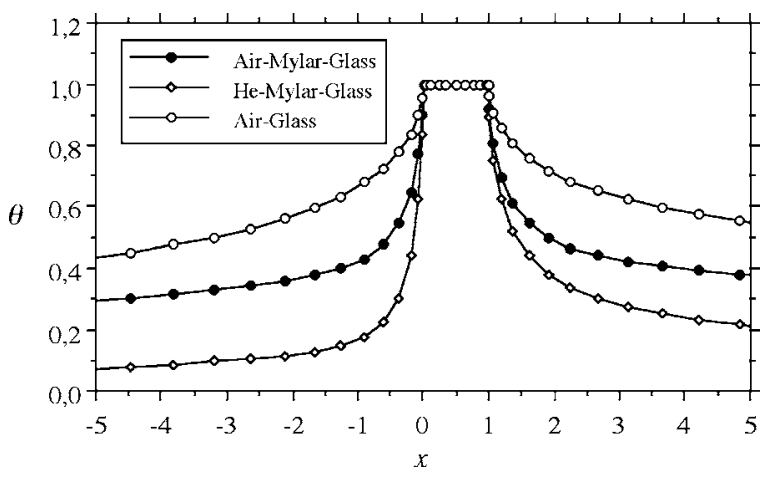

Fig. 10 Wall temperature distribution for several fluid/ sunstrate combinations

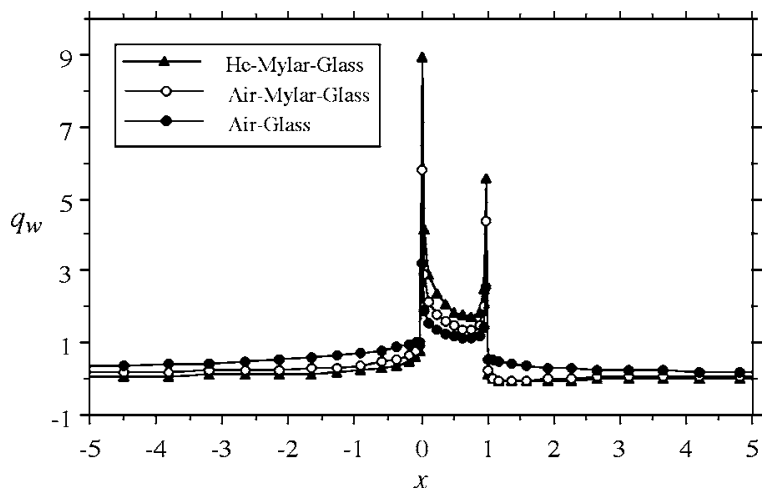

Fig. 11 Effect of the fluid/substrate configuration on the local flux for $\bar{\sigma}=30$ only entering into the consideration in the double-layer substrate configuration. To limit the number of cases considered, results for $\bar{\sigma}=30$ and $a=0.5$ are presented. The imposed frequency $\omega^{*}$ ranged from $10^{-6}$ to 10 . Typical dimensions of commercial films are $l_{f}$ $=0.2 \mathrm{~mm}$, so that $\sigma=30$ in air corresponds to $u_{\tau}=0.5 \mathrm{~m} / \mathrm{s}$, i.e., to a boundary layer with a free stream velocity of $\hat{u}_{\infty}=12 \mathrm{~m} / \mathrm{s}$.

The code was tested by investigating the exact 1D timedependent three-layer case of Bellhouse and Schultz [9] (F-S-F, with solid thickness $l_{f}$ and with the same flow on both sides of the solid slab). Figure 12 shows the relative amplitude of the heat transfer versus frequency for the case of air and water flow over glass. The reference amplitude is that of the quasi-steady case. The oscillations of the total heat transfer rate are damped with increasing frequency because the part transferred through the solid is filtered. This part is proportionally larger in air than in water flow and is increased by the effect of longitudinal diffusion in the fluid and in the solid (which is, of course, inherently neglected in the 1D solution of Bellhouse and Schultz). Figure 12 shows also that the attenuation of the frequency response of the full simulation, which takes axial diffusion into account, starts at a frequency which is about ten times lower than in the 1D exact solution.

The anemometer measures the total heat transfer from the film to the fluid and to the solid $Q=Q_{f F}+Q_{f S}$. In the unsteady regime, there cannot be an instantaneous balance between $Q_{f S}$ and $\left(Q_{S F}^{-}\right.$ $\left.+Q_{S F}^{+}\right)$as in the steady regime because the heat flux variations imposed at the solid surface by the velocity changes are filtered to variable degrees by the substrate, depending upon the distance to the film and the frequency.

The attenuation of the hot-film response is seen in Figs. 13 and 14 for the air-glass configuration. The cyclic variations of the relative transfer rates scaled with their time-mean values such as $Q_{f F} / \bar{Q}_{f F}$ (Fig. 13), $Q_{f S} / \bar{Q}_{f S}$, and the relative total flux $Q / \bar{Q}$ (Fig. 14) are shown versus time divided by the imposed period $P$ $=2 \pi / \omega^{*}$ for respectively a low $\left(\omega^{*}=10^{-3}\right)$ and high frequency $\left(\omega^{*}=3\right)$. One has $\omega^{*}=\sigma \omega^{+}$, where $\omega^{+}$is the frequency scaled with the shear and the viscosity, i.e., with the inner variables of a near wall turbulent flow. Thus, $\omega^{*}=3$ is quite high and corresponds to $\omega^{+}=0.1$, which is the frequency where the energy spectrum of the velocity is maximum near the viscous layer. This is also approximately $1 / 15$ of the Kolmogoroff frequency near the wall under these flow conditions. The low frequency $\omega^{*}=10^{-3}$ is nearly in the quasi-steady regime, wherein the transfer rates are in time equilibrium with the shear modulation. The real quasi-steady regime is difficult to reach, since the distance to which the heat diffuses during the period of oscillation is $\delta^{2} \approx \alpha_{S} T$, i.e., $\delta^{2} \approx\left(2 \pi / \omega^{*}\right)$ $\times\left(\alpha_{S} / \alpha_{F}\right) l_{f}^{2}$. The transfer through the substrate can only respond without time lag to velocity variations if $\delta \ll l_{f}^{e}$, i.e., if $\omega^{*}$ $\ll 2 \pi\left(l_{f} / l_{f}^{e}\right)^{2}\left(\alpha_{S} / \alpha_{F}\right)$. Since for air-glass combination $\alpha_{S} / \alpha_{F}$ $=0.017$ and $l_{f}^{e}=13 l_{f}$, for $\sigma=30$, this requires $\omega^{*} \ll 6 \times 10^{-4}$, a very small frequency indeed. Clearly, the air-glass combination is unfavorable for two reasons: first, because of the larger transfer through the substrate due to the large value of the conductivity ratio $k_{S} / k_{F}$, and, second, because $\alpha_{S} / \alpha_{F} \ll 1$, which results in considerable filtering of the variations of upstream and downstream transfers via the substrate. Similar computations indicate that the quasi-steady regime is reached for $\omega^{*} \ll 4.7$ (water/glass) and $\omega^{*}$ $\ll 9 \times 10^{-4}$ (He/mylar/glass or air/mylar/glass).

The frequency response of the direct transfer $Q_{f F} / \bar{Q}_{f F}$ is not significantly sensitive to the diffusivity and conductivity ratios. This can be seen in Fig. 13, which compares the cyclic modulation of the ideal Lévêque solution, the quasi-steady and highfrequency response of the hot film in air on a glass substrate. Figure 15 compares the direct transfer response of the air/glass with water/glass configurations for which it is recalled that the conductivity ratios vary by a factor $20 . Q_{f F} / \bar{Q}_{f F}$ is less modulated in air/glass but the difference is only a few percent. The modula- 

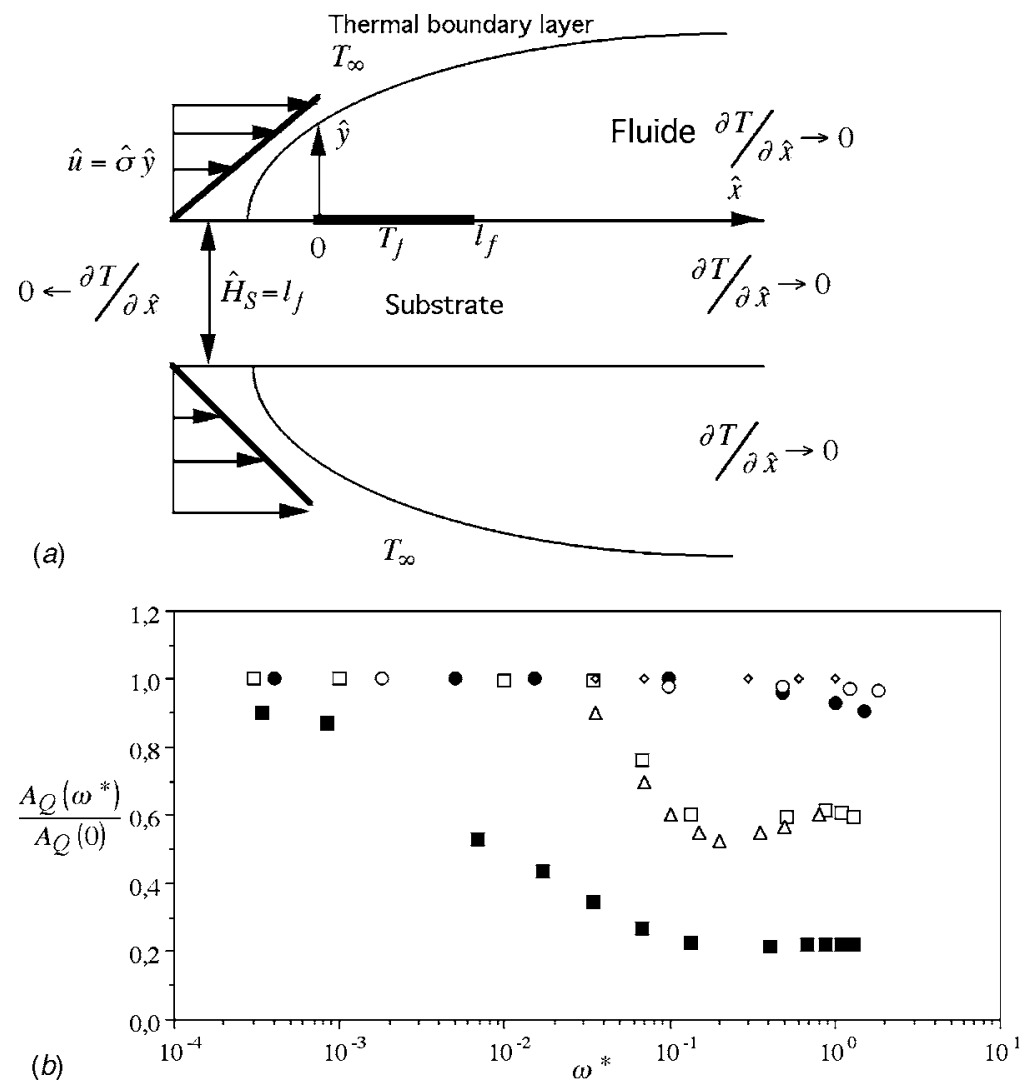

Fig. 12 (a) Problem investigated by Bellhouse and Schultz (1968). (b) Comparison with the present numerical results. $\square$ : Air with axial diffusion. $\square$ : Air without axial diffusion. $\bullet$ : Water with axial diffusion. $\bigcirc$ : Water without axial diffusion. $\triangle$ : Bellhouse and Schultz air. $\diamond:$ Bellhouse and Schultz water.

tion of the indirect transfer $Q_{f S} / \bar{Q}_{f S}$ is entirely frozen in the highfrequency regime as seen in Fig. 14, by comparing $Q_{f S} / \bar{Q}_{f S}$ in the quasi-steady $\omega^{*}=1.7 \times 10^{-4}$ and the high-frequency $\omega^{*}=3$ regimes. It can be easily shown that the amplitude of the global heat transfer $A_{Q}$ is the sum $A_{Q_{f S}}+A_{Q_{f F}}$ if the phase shifts can be neglected, which is approximately the case here. Since in the highfrequency regime $A_{Q_{f S}} \approx 0$, the amplitude of the global transfer decreases drastically by some $60 \%$ in the high-frequency regime. For low conductivity ratios as for water/glass configuration, the decrease of the amplitude $A_{Q_{f S}}$ is only $12 \%$, and the global transfer responds acceptably well in the high-frequency

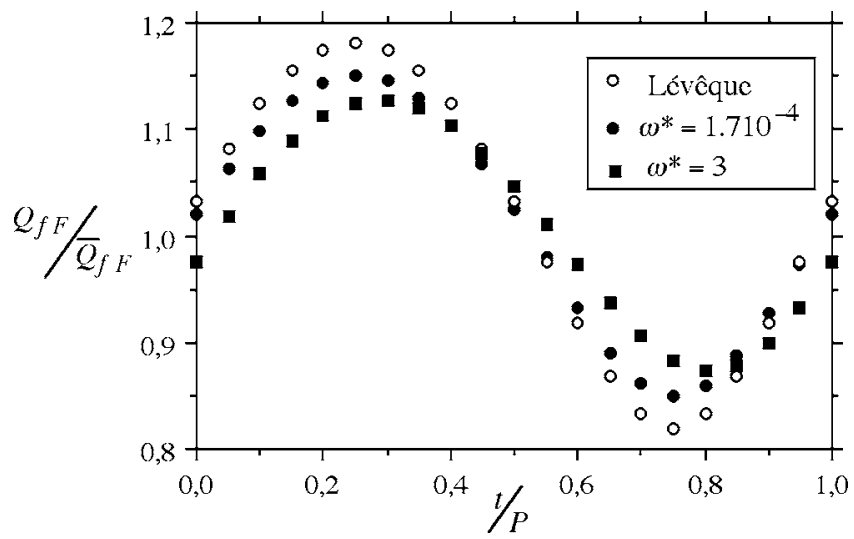

Fig. 13 Direct flux from the film to the fluid for air/glass case regime (Fig. 16).

Some values of the frequency response of the wall hot film are plotted in Fig. 17 for $\bar{\sigma}=30$. The amplitude of the global flux $A_{Q}$ is scaled with the amplitude in the quasi-steady regime $A_{Q}(\omega$ $=0)$ that was determined here at $\omega^{*}=10^{-6}$. The limiting value of the attenuation is $1 / 5$ when the mean flow parameter $\sigma=30$ in the air-glass case. This value is in reasonable agreement with the reduced turbulent intensity of the wall shear stress measured by some authors in air flows [18]. It is seen that the amplitude is already reduced by a factor of $1 / 2$ when $\omega^{*}=3 \times 10^{-4}$, while the thin insulating mylar film improves somewhat the response com-

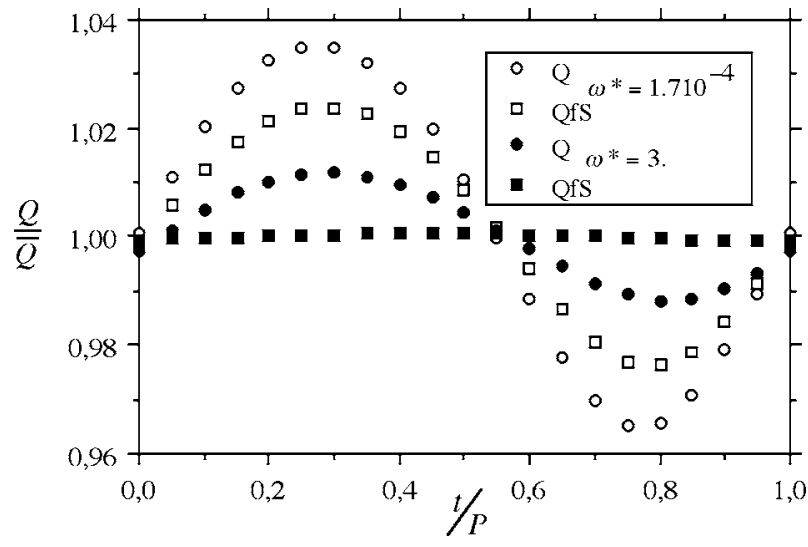

Fig. 14 Total flux and indirect flux from the film to the substrate for the air/glass configuration 


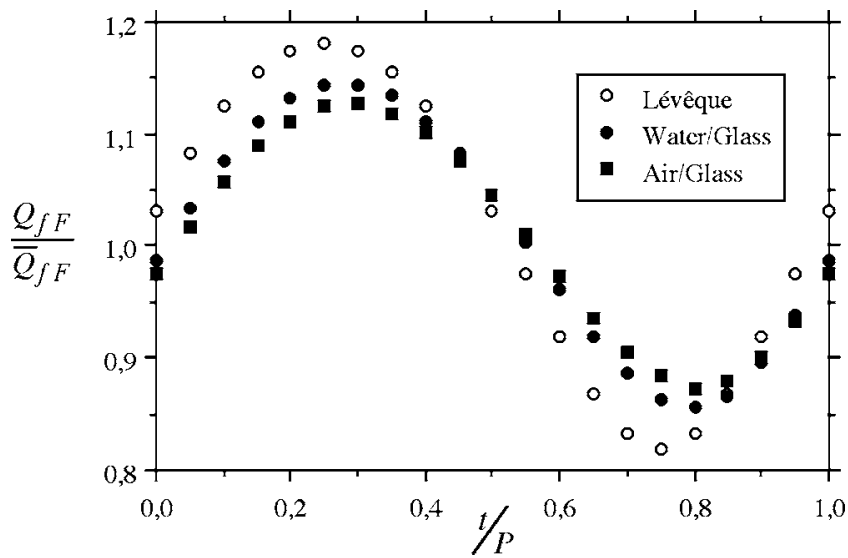

Fig. 15 Cyclic modulation of the direct flux for different configurations compared with the ideal Lévêque solution

pared with the air/glass case, but this improvement is far from being totally satisfactory. The thermal boundary layer itself has a finite frequency response owing to the finite transit time over the film $l_{f} / u_{\tau}$. But this frequency limit $\left(\omega^{*} \approx 1\right)$ is much higher than the limit imposed by the transfer via the substrate.

To show how severe the filtering effect of the substrate may be in the air/glass case, consider a turbulent boundary layer with $\hat{u}_{\infty}=12 \mathrm{~m} / \mathrm{s}$ and $\operatorname{Re}_{\delta}=5 \times 10^{4}$. The Kolmogoroff frequency is

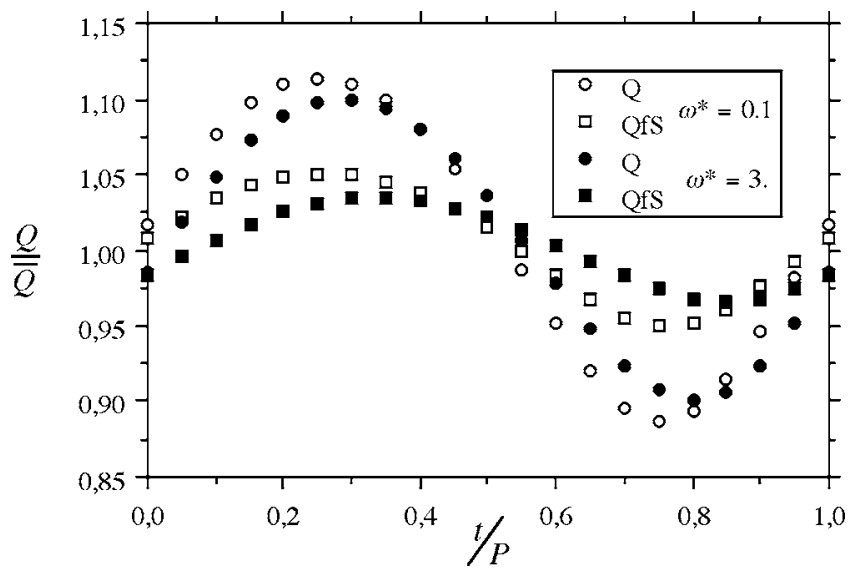

Fig. 16 Modulation of the total and indirect fluxes for the water/glass configuration

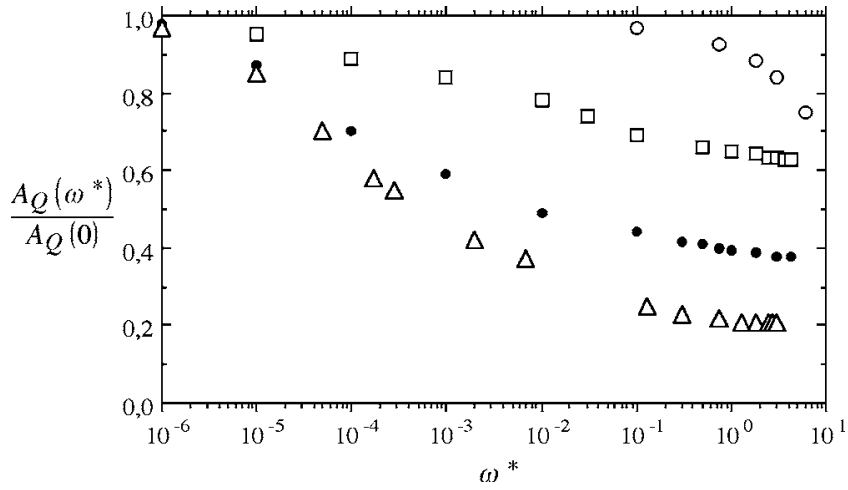

Fig. 17 Frequency response of the hot-film gage in different fluid/substrate configurations. $\bigcirc$ : Water-glass. $\square$ : Heliummylar-glass. $\bullet$ : Air-mylar-glass. $\triangle$ : Air-glass. roughly $5 \mathrm{kHz}$ in this case. The dimensionless frequency $\omega^{*}$ $=0.1$ at which the attenuation is $1 / 5$ is only $10 \mathrm{~Hz}$, i.e., nearly three orders of magnitude smaller than the Kolmogoroff frequency.

The comparison the water/glass with the He/mylar/glass reveals the predominance of the conductivity ratio in the dynamic response rather than the diffusivity ratio. The ratio of conductivities are respectively $k_{M y l a r} / k_{H e}=1, k_{\text {Glass }} / k_{H e}=5.2$, and $k_{\text {Glass }} / k_{\text {Water }}$ $=1.3$. The diffusivities vary considerably from a factor 4000 to 1000 with $\alpha_{\text {Glass }} / \alpha_{\text {Water }}=4190 \alpha_{\text {Mylar }} / \alpha_{\text {He }}$ and $\alpha_{\text {Glass }} / \alpha_{\text {Water }}$ $=1157 \alpha_{\text {Glass }} / \alpha_{H e}$. The distribution $A_{Q}\left(\omega^{*}\right) / A_{Q}(0)$ for the Hemylar-glass is below the water/glass configuration because $k_{\text {Glass }} / k_{H e}$ is higher than $k_{\text {Glass }} / k_{\text {Water }}$, but the large variations of the diffusivities do clearly not play a significant role in the heat transfer process.

The frequency response for the water/glass combination is reasonably acceptable. The attenuation is only $20 \%$ at $\omega^{*}=10$. The ratio $A_{Q}\left(\omega^{*}\right) / A_{Q}(0)$ is roughly 0.65 at the Kolmogoroff frequency $\omega_{K}^{*}=2 \pi f_{K}^{*}=60$ in a turbulent fully developed water channel flow with a Reynolds number based on the half-channel height of $10^{4}$. Thus wall shear stress measurements in a fully developed turbulent water channel flow by a hot film on glass can be reliably performed, except maybe at the smallest turbulent scales. Recall that the satisfactory frequency response of the case water/glass does not mean that there is no parasitic heat conduction to the substrate. On the contrary, the ratio $Q_{f S} / Q_{f F}$ varies between 1 and $3 / 5$ as seen in Fig. 5(a). Yet, this ratio reaches values as large as 10 in the case air/glass (Fig. 5(b)). The heat loss ratios of roughly $Q_{f S} / Q_{f F} \approx 1$ do not affect considerably the frequency response at least up to $\omega^{*}=1$.

\section{Conclusion}

A numerical investigation of heat transfer of wall-mounted hot film gages shows that heat transferred to the fluid via the substrate has important effects. For high values of the conductivity ratios $k_{S} / k_{F}$, the transfer from the film to the substrate may be considerably larger than the direct transfer to the fluid. For the air/glass combination, the transfer to the fluid through the substrate is about 10 times larger than the direct transfer from the film. Because of the hot wake, the downstream transfer from the substrate to the fluid is roughly half of the upstream value.

The importance of conduction into the substrate increases with decreasing velocity. A thin layer of material with low conductivity inserted between the film and substrate attenuates this effect.

The frequency response of the total heat transfer from the film, as measured by an anemometer set, is dominated by the filtering effect of the transient heat transfer via the substrate when $k_{F}$ $\ll k_{S}$ and $\alpha_{F} \gg \alpha_{S}$. For the air/glass combination, the response is attenuated as soon as $\omega^{*}>10^{-6}$. This limit is much lower than the cutoff frequency of the thermal layer.

There is a limit of the spanwise length $l_{f z}$ of the hot film probes to avoid spanwise averaging of the turbulence producing structures in the near wall region. According to Blackwelder and Haritonidis [19], this requires $l_{f z}^{+}=l_{f z}\left(u_{\tau} / \nu\right)<20$. The streamwise sensing length of some commercial wall hot-film gages is, $l_{f}=\frac{1}{8} l_{f z}$ to prevent both the spanwise conduction effects and to ensure twodimensionality. Therefore, the previous condition gives $l_{f}^{+}<2.5$. The corresponding Péclet number concerning the measurements of the wall shear stress and wall shear stress intensity in water is $\sigma=\operatorname{Pr} l_{f}^{+2}<44$. Clearly the axial diffusion through the leading and trailing edges singularities has to be taken into account under these circumstances. Note that the estimations given above constitute an upper limit. More careful measurements require at least $l_{f z}^{+}<10$ and $\sigma<10$. Neglecting the axial diffusion term in the energy equation when the Péclet number is small may generate serious errors in predicting the frequency response of the hot film. These points have to be taken into account in MEMS sensor designs. 


\section{Acknowledgment}

We are thankful to Professor Stefan Thynell for his continuous help in the editing process of this paper.

\section{Nomenclature}

$A_{q}=$ amplitude of the quantity $q$

$a_{q}=A_{q} / \bar{q}$, relative amplitude of the quantity $q$

$a=$ relative amplitude of the shear parameter

$h(\hat{x})=$ local heat transfer coefficient

$H=\int_{0}^{l} f(\hat{x}) d \hat{x}$, global heat transfer coefficient

$K=$ number of grid points over the hot film

$k=$ conductivity

$l_{f}=$ streamwise length of the hot film

$l_{f}^{e}=\left(Q / Q_{\text {Lévêque }}\right)^{3 / 2}$, dimensionless equivalent hotfilm length

$\mathrm{Nu}=H / k_{F}$, Nusselt number

$P=2 \pi / \omega^{*}$, dimensionless period of the imposed velocity oscillations

$\operatorname{Pr}=\nu / \alpha$, Prandtl number

$Q_{f F}=$ dimensionless direct total heat flux from the film to the fluid

$Q_{f S}=$ dimensionless heat flux from the film to the substrate

$Q_{\text {Lévêque }}=0.81 \sigma^{1 / 3}$, dimensionless direct heat flux over the film of the Lévêque solution.

$Q_{S F}^{-}=$dimensionless heat flux from the substrate to the fluid upstream of the hot film

$Q_{S F}^{+}=$dimensionless heat flux from the substrate to the fluid downstream of the hot film

$Q=Q_{f F}+Q_{f S}=\int_{\text {Film }} q_{w} d x$, dimensionless hot-film global heat transfer rate per unit span

$\bar{q}=$ time mean of the quantity $q$

$q^{+}=$quantity $q$ nondimensionalized with the shear velocity $u_{\tau}$ and viscosity $\nu$

$q_{w}=-(\partial \theta / \partial y)_{y=0}$, local heat flux

$q_{w}^{*}=-(\partial \theta / \partial y)_{y=0} \sigma^{-1 / 3}$, dimensionless local heat flux scaled with the shear parameter

$\operatorname{Re}_{\delta}=\hat{u}_{\infty} \delta / \nu$, turbulent boundary layer Reynolds number

$\operatorname{Re}_{f}=l_{f} u_{\tau} / \nu$, hot film Reynolds number

$T=$ temperature

$\hat{t}=$ dimensional time

$t=\left(\hat{t} / l_{f}^{2}\right) \alpha_{F}$, dimensionless time

$\hat{u}=$ dimensional velocity

$u=\hat{u} / u_{\tau}$, dimensionless velocity

$u_{\tau}=\sqrt{\tau / \rho_{F}}$, shear velocity

$\hat{x}=$ dimensional streamwise distance

$x=\hat{x} / l_{f}$, dimensionless streamwise coordinate

$\hat{y}=$ dimensional wall normal distance

$y=\hat{y} / l_{f}$, dimensionless wall normal coordinate

Greek symbols

$\alpha=$ diffusivity

$\delta=$ boundary layer thickness

$\eta=$ wall normal stretched coordinate

$\nu=$ kinematic viscosity

$\rho=$ density
$\sigma=\operatorname{Pr}\left(l_{f} u_{\tau} / \nu\right)^{2}$, shear parameter

$\bar{\sigma}=$ time-mean shear parameter

$\theta=\left(T-T_{\infty}\right) /\left(T_{f}-T_{\infty}\right)$, dimensionless temperature

$\tau=$ wall shear stress

$\hat{\omega}=$ dimensional angular frequency of the imposed oscillations

$=$ dimensionless angular frequency

$\omega^{+}=\hat{\omega}\left(\nu / u_{\tau}^{2}\right)$, imposed frequency in wall units.

$\xi=$ streamwise stretched coordinate

Abbreviations

$$
\begin{aligned}
F & =\text { fluid } \\
S & =\text { substrate } \\
\mathrm{WHF} & =\text { wall hot film }
\end{aligned}
$$

Subscripts

$$
\begin{aligned}
F & =\text { fluid } \\
S & =\text { substrate } \\
f & =\text { hot film } \\
\infty & =\text { bulk }
\end{aligned}
$$

\section{References}

[1] Tardu, S., 2001, "Active Control of Near Wall Turbulence by Local Unsteady Blowing," J. Fluid Mech., 43, pp. 217-253.

[2] Tardu, S., and Michelutti, L., 2004, "Microsystèmes Utilisés dans les Grands Écoulements," Microfluidique, S. Colin, ed., Hermès, Paris, pp. 349-389.

[3] Meunier, D., Tardu, S., Tsamados, D., and Bousssey, J., 2003, "Realization and Simulation of Wall Shear Stress Integrated Sensors," Microelectron. J., 34, pp. 1129-1136.

[4] Ling, S. C., 1963, "Heat Transfer from a Small Isothermal Spanwise Strip on an Insulated Boundary," ASME J. Heat Transfer, 85, pp. 203-235.

[5] Springer, S. G., and Pedley, T. J., 1973, "The Solution of Heat Transfer Problems by the Wiener-Hopf Technique. Leading Edge of a Hot-film," Proc. R. Soc. London, Ser. A, 333, pp. 347-362.

[6] Springer, S. G., 1974, "The Solution of Heat Transfer Problems by the WienerHopf Technique. Trailing Edge of a Hot-film," Proc. R. Soc. London, Ser. A, 337, pp. 395-412.

[7] Ackerberg, R. C., Patel, R. D., and Gupta, S. K., 1978, "The Heat/mass Transfer to a Finite Strip at Small Péclet Numbers,” J. Fluid Mech., 86, pp. 49-65.

[8] Lighthill, M. J., 1954, "The Response of Laminar Skin Friction and Heat Transfer to Fluctuations in Stream Velocity," Proc. R. Soc. London, 224, pp. $1-23$.

[9] Bellhouse, B. J., and Schultz, D. L., 1968, "The Measurement of Fluctuating Skin Friction in Air with Heated Thin-film Gauges," J. Fluid Mech., 32(2), pp. 675-680.

[10] Brison, J. F., Charnay, G., and Comte-Bellot, G., 1979, "Calcul des Transferts Thermiques Entre Film Chaud et Substrat Pour un Modèle à deux Dimensions," Int. J. Heat Mass Transfer, 22, pp. 111-119.

[11] Cole, K. D., and Beck, J. V., 1988, "Conjugated Heat Transfer from a Hot-film Probe for Transient Air Flow,” ASME J. Heat Transfer, 110, pp. 290-296.

[12] Moen, M. J., and Schneider, S. P., 1993, "The Effect of Sensor Size and Substrate Properties on the Performance of Flush-mounted Hot-film Sensors," Thermal Anemometry-1993, Stock, S. A. Sherif, A. J. Smits, J. Davidson, eds., ASME, New York, pp. 249-261.

[13] Pedleys, T. J., 1975, "A Thermal Boundary Layer in a Reversing Flow," J. Fluid Mech. 67, pp. 209-225.

[14] Fortuna, G., and Hanratty, T. J., 1971, "Frequency Response of the Boundary Layer on Wall Transfer Probes," Int. J. Heat Mass Transfer, 14, pp. 14991507.

[15] Kaiping, P., 1983, "Unsteady Forced Convective Heat Transfer from a Hot Film in Non Reversing and Reversing Shear Flow," Int. J. Heat Mass Transfer, 26(4), pp. 545-557.

[16] Raithby, G. D., and Torrance, K. E., 1974, "Upstream-weighted Differencing Schemes and Their Application to Elliptic Problems Involving Fluid Flow," Comput. Fluids, 2, pp. 191-206.

[17] Kalnay de Rivas, E., 1972, "On the Use of Nonuniform Grids in Finite Difference Equations," J. Comput. Phys., 10, pp. 202-210.

[18] Chambers, F. W., Murphy, H. D., and McEligot, D. M., 1983, "Laterally Converging Flow. Part 2. Temporal Wall Shear Stress," J. Fluid Mech., 127, pp. 403-428.

[19] Blackwelder, R. F., and Haritonidis, J. H., 1983, "Scaling of the Bursting Frequency in Turbulent Boundary Layers," J. Fluid Mech., 132, pp. 87-103. 